Universidade Tecnológica Federal do Paraná - UTFPR

Campus Ponta Grossa - Paraná - Brasil

ISSN: 1981-3686/v. 04, n. 02: p. 179-185, 2010

DOI: $10.3895 / \mathrm{S} 1981-36862010000200006$
Revista Brasileira de Tecnologia

Agroindustrial

\title{
AVALIAÇÃO DA CONTAMINAÇÃO POR AFLATOXINAS TOTAIS E ZEARALENONA EM VARIEDADES DE MILHO CRIOULO (Zea mays L.) ATRAVÉS DO MÉTODO IMUNOENZIMÁTICO ELISA
}

\section{CONTAMINATION EVALUTION FOR TOTAL AFLATOXINS AND ZEARALENONE IN VARIETIES OF LANDRACES MAIZE (Zea mays L.) THROUGH ELISA IMMUNOENZYMATIC METHOD}

\author{
Tatiana Roselena de Oliveira ${ }^{1}$; Ana Cláudia Barana ${ }^{2}$; \\ David de Souza Jaccoud-Filho ${ }^{3}$; Francisco Fanucchi Neto ${ }^{4}$. \\ ${ }^{1}$ Universidade Tecnológica Federal do Paraná - UTFPR - Ponta Grossa \\ tatianaroselena@yahoo.com.br \\ ${ }^{2}$ Universidade Estadual de Ponta Grossa - UEPG - Ponta Grossa anabarana@yahoo.com.br \\ ${ }^{3}$ Universidade Estadual de Ponta Grossa - UEPG - Ponta Grossa dj1002@ uepg.br \\ ${ }^{4}$ Universidade Estadual de Ponta Grossa - UEPG - Ponta Grossa fanucchi@ uepg.br
}

\begin{abstract}
Resumo
O milho que não sofreu intervenções genéticas, cultivado invariavelmente pela agricultura familiar é chamado de milho crioulo. O objetivo deste trabalho foi verificar a contaminação por aflatoxinas totais e zearalenona em variedades crioulas de milho do estado do Paraná. O método utilizado para a quantificação das micotoxinas foi o imunoenzimático ELISA. Foi possível quantificar uma contaminação por aflatoxinas em 70\% das amostras analisadas, em níveis que variaram de 1 a 2,6 ppb. Nenhuma das amostras analisadas quanto à contaminação por aflatoxinas totais estava acima do preconizado pela legislação brasileira. Das amostras analisadas quanto à contaminação por zearalenona, 70\% apresentou contaminação em níveis que variaram de 69,8 a 759,6 ppb. Os níveis de contaminação por zearalenona foram considerados altos comparados às legislações de alguns países. Por fim a contaminação por aflatoxinas totais nas amostras analisadas não se correlacionou com a contaminação por zearalenona nas mesmas amostras.
\end{abstract}

Palavras-chave: milho crioulo, aflatoxinas e zearalenona

\section{Introdução}

O milho (Zea mays) é uma planta originária da América Central, sendo que os povos indígenas foram os primeiros a utilizar este cereal, cultivando e selecionando diversas variedades primitivas de milho (GOODMAN, 1987).

Embora menos produtivas que os cultivares comerciais e praticamente não comporem as estatísticas da produção nacional de milho, as variedades locais, conhecidas como populações crioulas ou ainda landraces, apresentam uma importância significativa para a agricultura familiar no estado do Paraná (PATERNIARI et al., 2000). 
Como a maior parte dos cereais, o milho crioulo também pode ser afetado por fungos e seus metabólitos secundários (DINIZ, 2002). Os principais fungos encontrados em milho são pertencentes aos gêneros Fusarium, Aspergillus e Penicillium (MACHINSKI JUNIOR et al., 2001).

Esses fungos produzem metabólitos conhecidos como micotoxinas ou toxinas fúngicas que podem causar sérios danos à saúde humana e aos animais domésticos, além de provocar elevados prejuízos financeiros à exportação, uma vez que existem restrições impostas pelos países importadores de alimentos (LÁZZARI, 2000). As cinco mais importantes micotoxinas em alimentos são: aflatoxina, deoxinivalenol, nivalenol, zearalenona e fumonisina (DINIZ, 2002).

Em virtude da preocupação crescente com a qualidade dos alimentos produzidos, este trabalho tem por objetivos verificar a presença e quantificar as micotoxinas, aflatoxinas totais e zearalenona, em amostras de milho crioulo do estado do Paraná.

\section{Material e Métodos}

Para o presente estudo foram utilizadas dez variedades de milho crioulo originadas dos municípios de Rio Azul (PR), Prudentópolis (PR), Ponta Grossa (PR) e Pelotas (RS), cultivadas na fazenda escola da Universidade Estadual de Ponta Grossa na safra 2006/2007, utilizando um delineamento experimental em quatro blocos ao acaso.

As variedades utilizadas neste estudo foram: Nutricional $n{ }^{\circ} 61$, Grande $n{ }^{\circ} 52$, Palha Roxa $n$ ${ }^{\circ} 72$, Paiol n ${ }^{\circ} 49$, Branco $n$ 5, Asteca $n{ }^{\circ} 60$, Caiano $n{ }^{\circ} 63$, Roxo Índio I $n{ }^{\circ} 06$, Branco para palha $\mathrm{n}$ ${ }^{\circ} 69$ e Pérola ${ }^{\circ} 65$.

\section{Preparo das amostras}

As amostras foram moídas até uma granulometria de 20 mesh e quarteadas para tomada de amostra analítica. Foram utilizadas $50 \mathrm{~g}$ de cada amostra para a realização do teste.

\section{Determinação de micotoxinas}

As micotoxinas analisadas foram Aflatoxinas Totais e Zearalenona em duplicata. O método ELISA detecta e amplifica a reação antígeno-anticorpo pela ligação covalente entre enzimaanticorpo ou enzima-analito, cuja presença é determinada pela adição de enzima no substrato. A quantidade de substrato convertido a um dado tempo é indicativo da concentração original do composto a ser analisado. 


\section{Aflatoxinas Totais}

Utilizando-se 50 gramas da amostra, triturada e homogeneizada em blender em uma mistura de metanol e água $(80: 20 \mathrm{v} / \mathrm{v})$, por um minuto. Essa mistura é mantida em repouso por aproximadamente dois minutos, para a separação do material sólido, sendo o sobrenadante filtrado (mínimo 10mL), primeiramente em papel filtro qualitativo e após em papel filtro quantitativo. Esse sobrenadante constitui-se no extrato para a realização da análise. Os extratos são armazenados sob refrigeração, por no máximo 12 horas.

Utilizou-se o Kit de ELISA da Romer AgraQuant, Aflatoxinas Totais 1/20. A metodologia foi a disponibilizada pela ROMER LABS METHODS (2004).

Após a extração dos componentes da amostra, foi realizado o imunoensaio.

As absorbâncias das amostras foram lidas em uma leitora de microplacas Bio Tek, modelo ELX 800 com um filtro de $450 \mathrm{~nm}$. Comparando-se as absorbâncias dos padrões e das amostras chegou-se a concentração de aflatoxinas totais nas amostras.

\section{Zearalenona}

As amostras para a análise de zearalenona foram extraídas com a mesma metodologia empregada para a análise de aflatoxinas, exceto por uma diluição final da amostra em cinco vezes. Os extratos foram armazenados sob refrigeração, por no máximo 12 horas.

As amostras foram analisadas quanto à contaminação por Zearalenona pelo Kit de ELISA da Beacon Analytical Systems Inc, utilizando a metodologia disponibilizada pela Beacon Analyical Systems Inc.

As absorbâncias foram lidas em uma leitora de microplacas Bio Tek, modelo ELX 800 com um filtro de $450 \mathrm{~nm}$. Comparando-se as absorbâncias dos padrões e das amostras chegou-se na concentração de zearalenona nas amostras.

\section{Resultados e Discussão}

Nenhuma das dez amostras analisadas, quanto a concentração de aflatoxinas totais, estava fora dos padrões preconizados pela Resolução RDC - Anvisa no 274, de 15 de outubro de 2002 (BRASIL, 2002), que limita a concentração máxima de aflatoxinas totais nas amostras a 20 ppb.

Foi possível verificar ainda, que $70 \%$ das amostras estavam contaminadas por aflatoxinas totais em níveis de variaram entre 1,0 e 2,6 ppb.

Os valores de contaminação por aflatoxinas totais e zearalenona nas amostras analisadas estão apresentados na Tabela 1. 
Tabela 1 - Contaminação por aflatoxinas totais e zearalenona nas amostras analisadas em ppb.

\begin{tabular}{|c|c|c|}
\hline Variedades & Aflatoxinas totais (ppb) & Zearalenona (ppb) \\
\hline Nutricional $n{ }^{\circ} 61$ & $\mathrm{ND}$ a & $82,9 \mathrm{f}$ \\
\hline Grande n ${ }^{\circ} 52$ & $2,4 \mathrm{a}$ & $203,8 \mathrm{c}$ \\
\hline Palha Roxa ${ }^{\circ}{ }^{\circ} 2$ & $\mathrm{ND}$ a & $\mathrm{ND} h$ \\
\hline Paiol n ${ }^{\circ} 49$ & $1,0 \mathrm{a}$ & $333,9 \mathrm{~b}$ \\
\hline Branco n ${ }^{\circ} 57$ & $2,0 \mathrm{a}$ & $120,0 \mathrm{~d}$ \\
\hline Asteca $n{ }^{\circ} 60$ & $1,9 \mathrm{a}$ & $69,8 \mathrm{~g}$ \\
\hline Caiano ${ }^{\circ} 63$ & $2,6 \mathrm{a}$ & 759,6 a \\
\hline Roxo Índio I n ${ }^{\circ} 06$ & $1,2 \mathrm{a}$ & $105,7 \mathrm{e}$ \\
\hline Branco para palha n ${ }^{\circ} 69$ & $1,6 \mathrm{a}$ & ND h \\
\hline Pérola n ${ }^{\circ} 65$ & $\mathrm{ND} \mathrm{a}$ & $\mathrm{NDh}$ \\
\hline
\end{tabular}

Amostras com a mesma letra na mesma coluna não diferem estatisticamente através do teste de Tukey (p<0,05). ND. Níveis não detectáveis. LD - Aflatoxina: 1 ppb. LD - zearalenona: 55 ppb. LD: limite de detecção do método.

Os resultados apresentados são semelhantes aos apresentados por Caldas et al. (2002), que analisaram 33 amostras de milho em grão do Brasil e não detectaram aflatoxinas totais. E estão abaixo dos relatados por Machinski Júnior. et al. (2001), que detectaram a presença de aflatoxinas em 54,5\% das amostras de milho analisadas com níveis que variaram entre 6 e 1600 ppb. Vargas et al. (2001) também analisaram amostras de milho de diferentes regiões do Brasil e observaram contaminação de 38,3\% nas amostras com níveis médios de 9,4 ppb. Nas amostras analisadas para zearalenona a contaminação variou de 69,8 ppb a 759,6 ppb.

Verifica-se na Tabela 1 que houve diferença significativa $(\mathrm{p}<0,05)$ entre as amostras analisadas quanto a contaminação por zearalenona, não havendo esta diferença nas amostras analisadas quanto a contaminação por aflatoxinas totais.

Os resultados encontrados para a contaminação por zearalenona são semelhantes aos apresentados por diversos autores. Briones-Reyes; Gómez-Martinez e Cueva-Rolón (2007), que analisando 24 amostras de milho para consumo humano com origem no México, verificaram um índice de contaminação por zearalenona de 70\%. Marques (2007), analisando 47 amostras de subprodutos de milho para alimentação animal, obteve uma contaminação por zearalenona em $51 \%$ das amostras, em concentrações que variaram entre 100 e 5000 ppb. Janardhana; Raveesha e Shetty (1999) analisando a presença de ZEA em 197 amostras de milho encontraram uma incidência de 9,6\% com concentrações que variaram de 10 a 41 ppb.

Oliveira et al (2009), analisando amostras de milho crioulo no estado do Paraná quanto a contaminação por zearalenona, utilizando o método de cromatografia por camada delgada obtiveram resultados semelhantes aos escontrados neste estudo, com níveis de contaminação por zearalenona variando entre 64 e 640 ppm. 
O Brasil ainda não possui legislação para zearalenona, entretanto, sugere-se que os valores encontrados podem ser considerados altos, comparados aos limites permitidos pelas legislações de alguns países da ANVISA (BRASIL, 2010) e com a resolução em consulta pública (CP) (Tabela 2).

Tabela 2 - Limites permitidos (ppb) de contaminação por zearalenona em alguns países

\begin{tabular}{ccc}
\hline País & Limite permitido (ppb) & Produto \\
\hline Uruguai & 200 & Milho e cevada \\
Áustria & 50 & Ração animal \\
França & 200 & Cereais \\
Itália & 100 & Cereais e subprodutos \\
Romênia & 30 & Todos os alimentos \\
Rússia & 1000 & Café \\
Brasil (CP) & 400 & Milho não processado \\
\hline
\end{tabular}

Fonte: Legislação sobre micotoxinas CE 1881, 2006 e Brasil, 2010. CP: consulta pública.

Das amostras analisadas, 30\% estão acima do estipulado por países como a Uruguai e França, sendo portanto inadequadas para consumo nestes países.

Ainda entre as amostras analisadas, somente uma apresentou-se acima do estabelecido para milho não processado na Proposta de Resolução realizada pela ANVISA (BRASIL, 2010).

Na Tabela 3, é demonstrada a matriz de correlação entre a contaminação por aflatoxinas totais e zearalenona.

Tabela 3 - Matriz de correlação entre os dados de contaminação por aflatoxinas totais e zearalenona nas amostras analisadas

\begin{tabular}{lll}
\hline & Aflatoxinas Totais & Zearalenona \\
\hline Aflatoxinas Totais & 1 & 0,5623 \\
Zearalenona & 0,5623 & 1 \\
\hline
\end{tabular}

Através da Tabela 3 verifica-se que a correlação entre as variáveis foi de $R=0,5623$, não sendo considerada significativa $(\mathrm{p}<0,05)$. Sendo assim, a contaminação pelas duas micotoxinas analisadas não se correlacionaram. Portanto, os fatores que favorecem a contaminação por um tipo de micotoxina, podem não interferir na contaminação por outra.

\section{Conclusões}

A incidência de aflatoxinas totais ocorreu em $70 \%$ das amostras, mas todas estavam abaixo do limite permitido pela legislação brasileira. A análise de contaminação por zearalenona revelou a contaminação dos grãos em níveis que variaram entre 66,9 e 759,3 ppb, sendo encontrada em $70 \%$ das amostras analisadas. A incidência das duas micotoxinas analisadas não se correlacionaram significativamente, não ocorrendo portanto, inibição ou favorecimento da contaminação de uma micotoxina na presença de outra. 
Conclui-se ainda, que os níveis de contaminação por zearalenona foram altos, quando comparados às legislações de alguns países. A implementação de leis que especifiquem a contaminação máxima por zearalenona em grãos para consumo é de grande importância, evitandose assim grãos excessivamente contaminados, o que pode prejudicar a saúde humana.

\begin{abstract}
The corn that was not genetic interventions and invariably it is grown on family farms corn is called Landrace. The aim of this study was to investigate the contamination by aflatoxins and zearalenone in total landraces of maize in the state of Parana. The method used for quantification of mycotoxins was the ELISA immunoassay. It was possible to quantify aflatoxin contamination in $70 \%$ of samples, at levels ranging from 1 to $2.6 \mathrm{ppb}$. None of the samples analyzed for total aflatoxin contamination was above the recommended by Brazilian legislation. Of the samples analyzed for contamination by zearalenone, $70 \%$ showed contamination levels ranging from 69.8 to $759.6 \mathrm{ppb}$. The levels of contaminatios by zearalenone were considered high when compared to the laws of some conuntries. Finally, the total aflatoxin contamination in the samples was not correlated with the contamination by zearalenone in the same samples.
\end{abstract}

Key-Words: landrace maize, aflatoxins, zearalenone

\title{
5. Referências
}

BEACON ANALYTICAL SYSTEMS INC. Protocolo de análise de kit ELISA para zearalelona, 2006.

BRASIL. Ministério da Agricultura. Agência Nacional de Vigilância Sanitária. Resolução - RDC no 274 , de 15 de outubro de 2002. Brasília. 2002.

BRASIL. Ministério da Agricultura. Agência Nacional de Vigilância Sanitária. Proposta de Resolução. Brasília. 2010

BRIONES-REYES, D.; GÓMEZ-MARTINEZ, L.; CUEVA-ROLÓN, R. Zearalenone contamination in corn for human consumption in the state of Tlaxcala, Mexico. Food Chemistry, v. 100, n. 02, p. 693-698, 2007. DOI: 10.1016/j.foodchem.2005.10.027.

CALDAS, E. D.; SILVA, S. C.; OLIVEIRA, J. N. Aflatoxinas e ocratoxina A em alimentos e riscos para a saúde humana. Revista Saúde Pública, v. 36, n. 3, p. 319- 323, 2002. DOI:10.1590/S0034-89102002000300010.

DINIZ, S. P. S. S. Micotoxinas, 1 ed, Campinas: Livraria e Editora Rural, 2002.

GOODMAN, M. História e origem do milho. In PATERNIARI, E. (ed) Melhoramento e produção de milho no Brasil. Fundação Cargil, 1987, 650 p.

JANARDHANA, G. R K.; RAVEESHA, A.; SHEKAR SHETTY, H. Mycotoxin Contamination of Maize Grains Grown in Karnataka (Índia). Food and Chemical Toxicology, v. 37, n. 8, p. 863-868, 1999. 868, 1999. DOI:10.1016/S0278-6915(99)00067-8.

LAMIC. Legislação sobre micotoxinas CE 1881/2006, disponível em: <http://www.lamic.ufsm.br/legislacao.html> . Acesso em 30 abr. 2010.

LAZZARI, F. Rações e micotoxinas. In: SCUSSEL, V. M. (Org) Atualidades em Micotoxinas e Armazenagem de Grãos. Florianópolis, VMS, 2000, 382p.

MACHINSKI, J. M.; SOARES, L. M. V.; SAWAZAKI, E.; BOLONHEZI, D.; CASTRO, J. L.; BORTOLLETO, N. Aflatoxins, ochratoxin A and zearalenone in Brazilian corn cultivars. Journal of the Science of Food and Agriculture, v. 81., n. 10, p. 1001-1007, 2001.DOI: 10.1002/jsfa.882. 
MARQUES, P. J. L.; Avaliação de aflatoxinas e zearalenona em quirera (canjiquinha de milho) na região dos Campos Gerais. 96 f. Dissertação (Mestrado em Ciência e Tecnologia de Alimentos) - Universidade Estadual de Ponta Grossa, Ponta Grossa, 2007.

OLIVEIRA, T. R.; JACCOUD-FILHO, D.; HENNEBERG, L.; MICHEL, M. D.; DEMIATE, I. M.; PINTO, A. B. T.; MACHINSKI, J. M.; BARANA, A. Maize (Zea Mays L) landraces from the southern region of Brazil: contamination by Fusarium sp, zearalenone, physical and mechanical characteristics of the kernels. Brazilian Archives of Biology and Technology, Curitiba, v. 52, ${ }^{\circ}{ }^{\circ}$ spe, p. 11-16, 2009.

ROMER LABS METHODS. Protocolo de análise de kit ELISA para aflatoxinas totais 1/20. Sisngapore, 2004.

VARGAS, E. A.; PREIS, R. A.; CASTRO, L.; SILVA, C. M. G. Co-occurrence of aflatoxins B1, B2, G1 e G2 zearalenone and fumonisin in Brazilian corn. Food and Additives Contaminants, Ireland, v. 18, n. 11, p. 981-986, 2001. DOI:10.1080/02652030110046190.

Dados dos autores

Nome completo: Tatiana Roselena de Oliveira

Filiação institucional: Universidade Tecnológica Federal do Paraná

Departamento: Coordenação do Curso Superior de Tecnologia em Alimentos

Função ou cargo ocupado: Professor

Titulação: Mestre em Ciência e Tecnologia de Alimentos

Endereço: Rua Sebastião Osório Martins, 7. Jardim Vila Velha. Ponta Grossa - Paraná - Brasil. CEP: 84030-457

Telefone para contato: (42) 3226-6096 ou (42) 9921-5636

e-mail: tatianaroselena@yahoo.com.br

Nome completo: Ana Claudia Barana

Filiação institucional: Universidade Estadual de Ponta Grossa

Departamento: Engenharia de Alimentos

Função ou cargo ocupado: Professor

Titulação: Doutor

Endereço: Departamento de Engenharia de Alimentos, Universidade Estadual de Ponta Grossa, Av. General Carlos Cavalcanti, 4748 - CEP 84.030-900 - Ponta Grossa - Paraná - Brasil.

Telefone para contato: (42) 3220-3268 e-mail: anabarana@yahoo.com.br

Nome completo: David de Souza Jaccoud-Filho

Filiação institucional: Universidade Estadual de Ponta Grossa

Departamento: Fitotecnia e Fitossanidade

Função ou cargo ocupado: Professor

Titulação: Doutor

Endereço: Departamento de Fitotecnia e Fitossanidade, Universidade Estadual de Ponta Grossa, Av. General Carlos Cavalcanti, 4748 - CEP 84.030-900 - Ponta Grossa - Paraná - Brasil.

Telefone para contato: (42) 3220-3086e-mail: dj1002@ uepg.br

Nome completo: Francisco Fanucchi Neto

Filiação institucional: Universidade Estadual de Ponta Grossa

Departamento: Análises Clínicas

Função ou cargo ocupado: Professor

Titulação: Doutor

Endereço: Departamento de Análises Clínicas, Universidade Estadual de Ponta Grossa, Av.

General Carlos Cavalcanti, 4748 - CEP 84.030-900 - Ponta Grossa - Paraná - Brasil.

Telefone para contato: (42) 3220-3113 e-mail: fanucchi@uepg.br 